\title{
Predictors of Radiation Pneumonitis in Patients with Lung Cancer after Chemoradiotherapy
}

\author{
Wafaa Ali Hassan*, Mostafa Abd-Elwaness \\ Chest Department, Oncology Institute, Assiut University, Assiut, Egypt \\ Email: "wafaagadallah@yahoo.com
}

Received 10 February 2014; revised 10 March 2014; accepted 17 March 2014

Copyright (C) 2014 by authors and Scientific Research Publishing Inc.

This work is licensed under the Creative Commons Attribution International License (CC BY). http://creativecommons.org/licenses/by/4.0/

(c) (i) Open Access

\begin{abstract}
The reported incidence of radiation pneumonitis (RP) has varied widely in clinical studies ranging from $0 \%$ to $54 \%$. This wide range is probably the result of differences in its precipitating factors. This study aimed to find out the predictive factors of RP in patients with NSCLC who were treated with concurrent chemotherapy and radiotherapy (CCRT). We prospectively studied 76 lung cancer patients who were treated with CCRT consecutively between January 2011 and May 2013 in the Department of Radiation Oncology at Cancer Institute, Assiut University. Radiographic images, pulmonary function tests and symptom assessment were used for the diagnosis of RP. Of the studied 76 patients, 25 patients developed RP (33\%), 15 of them developed grade 2 RP, 8 patients developed grade 3 RP, 1 patient developed grade 4 toxicity, and 1 patient developed grade 5 toxicity. In multivariate analysis, FEV1 value $(p=0.000)$ and chronic obstructive pulmonary disease (COPD) $(p=0.012)$ were the most significant factors associated with RP. Pretreatment FEV1 value and COPD are useful indicators for predicting RP in NSCLC patients treated with CCRT. Pretreatment of pulmonary function and base-line pulmonary disease is critical for patients' well being after chemoradiotherapy.
\end{abstract}

\section{Keywords}

NSCL, RP, CCRT, Pulmonary Function Test

\section{Introduction}

Radiation pneumonitis (RP) is one of the major dose-limiting toxicities in patients receiving radiotherapy for

"Corresponding author.

How to cite this paper: Hassan, W.A. and Abd-Elwaness, M. (2014) Predictors of Radiation Pneumonitis in Patients with Lung Cancer after Chemoradiotherapy. Open Journal of Respiratory Diseases, 4, 27-33. 
lung cancer [1]. Higher radiation doses will not only improve local control rate but also increase the incidence of treatment related toxicity [2] [3]. The reported incidence of radiation pneumonitis has varied widely in clinical studies ranging from $0 \%$ to $54 \%$ [4]. This wide range is probably the result of differences in the precipitating factors as total radiation doses, number of fractions and fraction dose, and the differences in associated chemotherapy regimens. Clinical manifestations of RP are usually nonproductive cough, mild fever and dyspnea on exertion. Besides these non-specific symptoms, several other factors such as difference in radiation techniques and variable reporting methods can contribute to the variability of the RP incidence [4]-[8]. Although most patients with mild RP recover spontaneously, some with severe RP suffer from bothering symptoms that can lead to poor quality of life and in a few cases to death. Lung cancer patients, who frequently suffer from underlying chronic obstructive pulmonary disease (COPD), present a major population where radiotherapy (RT) faces this challenge. RT must be carefully and conservatively planned for such patients to minimize comorbidities and complications related to surrounding lung injury [9].

At present, there is no generally accepted means to predict the individual patient's risk of developing radiation pneumonitis morbidity accurately even though dosimetric assessment of radiation pneumonitis has been studied extensively [1]-[5]. This study aimed to find out the predictive factors of RP in patients with NSCLC who were treated with CCRT.

\section{Patients and Methods}

We prospectively reviewed the medical and radiation records of lung cancer patients who were consecutively treated with concurrent chemotherapy and radiotherapy between January 2011 and May 2013 in the Department of Radiation Oncology at Cancer Institute, Assiut University. Patients were included if they had newly diagnosed and pathologically confirmed NSCLC and clinically staged as IIIa or IIIb, treated with CCRT. Radiographic images, PFTs and symptom assessment were used for determining the occurrence of RP. Our study was performed in accordance with the guidelines of the institutional review board of Faculty of Medicine of Assuit University that approved the study.

\section{Radiation Techniques and Radiation Parameters}

Patients were positioned in the treatment position (generally supine with arms above their heads) Radiotherapy was planned using standard 3D conformal techniques for all patients $>95 \%$ of the planning target volume receiving the prescribed dose. Radiation therapy was administered using photon beams, with energy between 6 and $15 \mathrm{MV}$. Target volume consisted of the original and boost volumes if the radiation dose were beyond $50 \mathrm{~Gy}$. Original volume was based on a planning CT scan taken before chemotherapy, and included primary lesion, any grossly involved nodal sites, plus ipsilateral hilum, and mediastinum with a margin of $2 \mathrm{~cm}$. Even if the primary tumor was in the lung periphery, only one radiation field was used to cover it and the mediastinum. The mean lung dose, lung volume irradiated to 20 Gy (V20), and volume of lung irradiated to 30 Gy (V30) were used as dosimetric parameters to estimate the lung volume irradiated. The right lung and the left lung were contoured separately and then taken into consideration as a single structure called "total lung," which was defined, as in the study of Graham et al. [4]: (Right lung + Left lung) - PTV. All treatment plans were approved if conventional dosimetric lung constraints were respected: V20 of 35\% or lower, V30 of $18 \%$ or lower, and mean lung dose of 20 Gy or lower [10].

Dose limitation was defined as follows: the V20 of lung less than $31 \%$, the V55 of esophagus less than $50 \%$, the V40 of heart less than $40 \mathrm{~Gy}$, and the maximum dose administered to the spinal cord was $40 \mathrm{~Gy}$. The concurrent chemotherapy consisted of Cisplatin, Gemcitabine or Navilbin [9].

\section{Evaluation of RP}

All patients were examined by their treating radiation oncologists weekly during radiotherapy and 4 - 6 weeks after completion of radiotherapy. The patients were then followed every 3 months unless they had symptoms that required immediate examination or intervention. Radiographic examination by chest X-ray or CT was performed at each follow-up visit after completion of chemoradiotherapy. If patients had symptoms, such as fever, cough or shortness of breath, they would be required to have an immediate examination or intervention. A diagnosis of RP was based on clinical symptoms and radiographic infiltrate changes corresponding to the radiation 
portal observed during concurrent chemoradiotherapy, within the first 6 months after treatment and in the absence of any other likely cause [10].

RP induced by chemoradiotherapy was diagnosed by the history of chemotherapy after radiotherapy, clinical presentation and radiographic abnormalities including ground-glass opacity, attenuation, or consolidation changes within the radiation field, plus that radiographic examination of the thorax before showed no radiation pneumonitis. RP was graded according to the National Cancer Institute's Common Terminology Criteria for Adverse Events (CTC) version 3.0 [11] as follows: Grade 1 pneumonitis was asymptomatic and diagnosed by radiographic findings only; Grade 2 pneumonitis was symptomatic but did not interfere with daily activities; Grade 3 pneumonitis was symptomatic and interfered with daily activities or required administration of oxygen to the patient; Grade 4 pneumonitis required assisted ventilation for the patient; and Grade 5 pneumonitis was fatal. Informed consent was obtained from all the subjects.

\section{Evaluation of Pulmonary Function}

Standard spirometric parameters were determined using a model Pulmonary Function Test Horizon Systems Spirometer (Sensor Medics, Italy). Spirometry was performed at least three times in each patient during each examination and as a measure of reproducibility at least two of the tests had to be within $5 \%$ of each other. The pulmonary function test data were described as a percentage of predicted values. The lung transfer factor for CO (DLCO) was measured with the single breath technique 2235. DLCO values measured while breathing room air were corrected for hemoglobin concentration [12].

\section{Statistical Analysis}

The following clinical parameters were considered: gender, age, smoking and history of COPD, induction chemotherapy, concurrent chemotherapy regimens, and forced expiratory volume in 1 second (FEV1). Pearson ChiSquare test was performed to compare clinical parameters between patients who developed RP and those who did not. Univariate and multivariate logistic regression analysis were performed to evaluate data associated with RP. All statistical tests were 2-sided and $\mathrm{P} \leq 0.05$ was considered statistically significant.

\section{Results}

Of the studied 76 patients, twenty five patients developed RP (33\%), of which 15 developed grade 2 RP, 8 patients developed grade 3 RP, 1 patient developed grade 4 toxicity, and 1 patient developed grade 5 toxicity. All patients received concurrent chemotherapy, The chemotherapy regimens were Cisplatin, Gemcitabine or Navilbin. There were 52 men and 24 women with a median age of 65 years (range, 45 - 81 years). Median follow-up was 8 months. RP occurred between 9 week and 16 week (median, 8 weeks) from start of treatment. Cancer stage was determined in all patients as per the 2006 Joint Committee on Cancer guidelines. There were reduced mean values for FVC, FEV1, and significantly reduced FEV1\% (40\% vs. 63\%) $(\mathrm{P}=0.000)$ (Table 1) in patients who developed RP related to CCRT. Univariate analysis showed that there was no significant difference between the clinical parameters (sex, age, smoking history, site, stage), histopathology, comorbidities other than COPD and chemotherapy regimen of patients with and without RP) (Tables 1-4). The presence of COPD (Table 4) was also significantly higher with RP $(72 \%$ vs. $49 \%)(\mathrm{P}=0.001)$. The results of the final multivariable model suggested that FEV1 and COPD were significant predictors of RP ( $\mathrm{P}=0.03$ and 0.01 , respectively).

\section{Discussion}

Radiation pneumonitis takes place usually within 1-6 months after completion of radiation therapy [13] [14]. The diagnosis of radiation pneumonitis is established by clinical symptoms, a history of radiotherapy, or radiographic evidence. The treatment for radiation pneumonitis largely includes oral or intravenous steroids, oxygen, antibiotics and sometimes, assisted ventilation. The reported incidences of radiation pneumonitis were inconsistent because of inconsistencies in the criteria used to define radiation pneumonitis, heterogeneity in patient populations, and differences in treatment regimens and radiotherapy techniques [15] [16]. Radiation pneumonitis is the most common toxicity after radiotherapy for thoracic tumors and can have substantial influence on the morbidity and mortality of patients with lung cancer. Therefore, it is essential to find out the predictive factors for the development of RP to reduce the incidence of RP. Many studies reported that radiation dose and irradiated 
Table 1. Patient characteristics and pulmonary function data in patients with and without RP.

\begin{tabular}{ccccc}
\hline & With RP & Without RP & & P value \\
& $(\mathbf{n = 2 5 )}$ & $(\mathbf{n = 5 1 )}$ & Univariate & Multivariate \\
\hline Age (ys) & & & & 0.123 \\
$>60$ & $20(80 \%)$ & $43(84 \%)$ & 0.341 & 0.342 \\
$<60$ & $5(20 \%)$ & $7(16 \%)$ & 0.876 & 0.473 \\
Sex & & & & 0.07 \\
Male & $23(92 \%)$ & $47(92 \%)$ & 0.451 & 0.09 \\
female & $2(8 \%)$ & $4(8 \%)$ & 0.234 & 0.03 \\
Smoking history & $21(84 \%)$ & $46(92 \%)$ & 0.06 & 0.081 \\
Pretreatment PFT & & & & 0.072 \\
FEV1 (\% predicted) & $40.66 \pm 8.24$ & $63.16 \pm 5.24$ & 0.000 & 0.081 \\
FVC (\% predicted) & $60.82 \pm 21.23$ & $58.26 \pm 11.33$ & 0.761 & 0.345 \\
FEV1/FVC & $59.00 \pm 12.72$ & $62 \pm 14.23$ & 0.671 & \\
DLCO (\% predicted) & 49 & 55 & & \\
\end{tabular}

Table 2. Clinical stage, site of tumor and histopathology in patients with and without RP.

\begin{tabular}{ccccc}
\hline & With RP (n = 25) & Without RP (n = 51) & \multicolumn{2}{c}{ P value } \\
\hline Clinical stage & & & Univariate & Multivariate \\
III a & $9(36 \%)$ & $17(33 \%)$ & 0.453 & 0.082 \\
III b & $16(64 \%)$ & $34(67 \%)$ & 0.564 & 0.571 \\
Site of the tumor & & & 0.123 \\
Upper lobe & $10(40 \%)$ & $20(39 \%)$ & 0.341 & 0.342 \\
Lower lobe & $15(60 \%)$ & $31(61 \%)$ & 0.876 & 0.521 \\
Central tumor & $18(72 \%)$ & $43(84 \%)$ & 0.435 & 0.645 \\
Peripheral tumor & $7(23 \%)$ & $8(16 \%)$ & 0.657 & 0.473 \\
Histopathology & & & & 0.07 \\
Squamus cell & $13(52 \%)$ & $23(45 \%)$ & 0.451 & 0.987 \\
carcinoma & & $10(19 \%)$ & 0.234 & 0.561 \\
Adenocarcinoma & $5(20 \%)$ & $18(36 \%)$ & & \\
Large cell & $7(28 \%)$ & & & \\
\hline
\end{tabular}

Table 3. Radiation dose and chemotherapy regimen in patients with and without RP.

\begin{tabular}{|ccccc} 
& $\begin{array}{c}\text { With RP } \\
(\mathbf{n}=\mathbf{2 5})\end{array}$ & $\begin{array}{c}\text { Without RP } \\
(\mathbf{n}=\mathbf{5 1})\end{array}$ & Univariate & Multivariate \\
\hline $\begin{array}{c}\text { Mean radiation dose (GY) } \\
\mathbf{2 0}\end{array}$ & & & & \\
$\mathbf{3 0}$ & $20(80 \%)$ & $40(78 \%)$ & 0.453 & 0.082 \\
& $5(20 \%)$ & $11(22 \%)$ & 0.564 & 0.571 \\
\hline
\end{tabular}

lung volumes are closely associated with the risk of RP. Several DV parameters have been proposed as predictive factors for RP but still there is no consensus about which parameters are ideal for predicting the RP. The incidence of RP is 33\% (25/76) in our study, which was more than that reported by Yom (11) using IMRT (12\%), and similar to other results using conventional radiotherapy [16] [17]. Perhaps this is because IMRT techniques, which had high conformity and spared more normal lung from irradiation, and therefore may have induced a lower rate of severe radiation pneumonitis. In our study, the patient population is quite homogeneous compared with most published studies: All patients had Stage III NSCLC, and received concurrent chemotherapy and 
Table 4. Co morbidities associated with RP.

\begin{tabular}{ccccc}
\hline & $\begin{array}{c}\text { With } \mathbf{R P} \\
\mathbf{( n = 2 5 )}\end{array}$ & $\begin{array}{c}\text { Without RP } \\
(\mathbf{n}=\mathbf{5 1})\end{array}$ & Univariate & Multivariate \\
\hline COPD & $18(72 \%)$ & $26(49 \%)$ & 0.001 & 0.01 \\
DM & $4(16 \%)$ & $7(13 \%)$ & 0.341 & 0.123 \\
stroke & $3(12 \%)$ & $5(10 \%)$ & 0.098 & 0.091 \\
OSAS & $2(8 \%)$ & $3(6 \%)$ & 0.087 & 0.765 \\
\hline
\end{tabular}

conventional radiotherapy. The homogeneity of demographic data in the study allowed us to focus on our studied factors.

There are many reported studies [18]-[20] in which the risks of radiation pneumonitis were found to be associated with a variety of clinical parameters. Sex, age, smoking history, pre-existing pulmonary disease, performance score and pulmonary function before radiotherapy have been reported to affect the risk for radiation pneumonitis [20] [21]. It also has been reported [22] [23] that chemotherapy, particularly when combined with thoracic radiation therapy, was associated with an increased risk for radiation pneumonitis. However, in our study, we only found that COPD and FEV1 were significantly associated with RP, suggesting that the pulmonary function before radiotherapy and base-line pulmonary disease is critical for patients' well being after chemoradiotherapy. Our findings are consistent with that of Robnett [9] and Rancati [6]. In addition, univariate analysis showed that there was no significant difference between the clinical parameters (sex, age, smoking history, site, stage), histopathology, comorbidities other than COPD and chemotherapy regimen of patients with and without RP.

One possible explanation for this result is the small sample size in this study. The discrete use of individualized radiotherapy was another factor. If a patient has COPD and therefore has poor lung function, it is recommended to modify the treatment plan and restrict the DV parameters than conventionally planned cases. This consideration might have compensated for the RP susceptibility of these vulnerable patients.

Regarding to pulmonary function, there were significant reductions in pretreatment pulmonary function in cases that developed RP related to CCRT particularly FEV1. Many investigators showed the decline in the PFT, and its correlation with the symptoms. Hope et al. [15] reported the change of pulmonary function in patients with long term disease free survival. Inoue et al. [24] reported similar result that showed significant reduction in PFT at 6 months after RT which continued beyond 1 year. We separately evaluated the PFT changes before the start of treatment. Lack of repeating PFTs after CCRT and to correlate the degree of pulmonary functional changes with the severity of RP and age adjustment were limitations in this study. In spite of these limitations, we can assume that pretreatment FEV1 and the association of COPD could predict the development of RP after CCRT in lung cancer. This needs to be explored with forthcoming studies.

\section{Conclusion}

Pretreatment FEV1 value and COPD are useful indicators for predicting RP in NSCLC patients treated with CCRT. Pretreatment of pulmonary function and base-line pulmonary disease is critical for patients' well being after chemoradiotherapy.

\section{References}

[1] Hernando, M.L., Marks, L.B., Bentel, G.C., et al. (2001) Radiation-Induced Pulmonary Toxicity: A Dose-Volume Histogram Analysis in 201 Patients with Lung Cancer. International Journal of Radiation Oncology * Biology * Physics, 51, 650-659. http://dx.doi.org/10.1016/S0360-3016(01)01685-6

[2] Yorke, E.D., Jackson, A., Rosenzweig, K.E., et al. (2002) Dose-Volume Factors Contributing to the Incidence of Radiation Pneumonitis in Non-Small-Cell Lung Cancer Patients Treated with Three-Dimensional Conformal Radiation Therapy. International Journal of Radiation Oncology * Biology * Physics, 54, 329-339. http://dx.doi.org/10.1016/S0360-3016(02)02929-2

[3] Kwa, S.L., Theuws, J.C., Wagenaar, A., et al. (1998) Evaluation of Two Dose-Volume Histogram Reduction Models 
for the Prediction of Radiation Pneumonitis. Radiotherapy \& Oncology, 48, 61-69. http://dx.doi.org/10.1016/S0167-8140(98)00020-6

[4] Graham, M.V., Purdy, J.A., Emami, B., et al. (1999) Clinical Dose-Volume Histogram Analysis for Pneumonitis after 3D Treatment for Non-Small Cell Lung Cancer (NSCLC). International Journal of Radiation Oncology * Biology * Physics, 45, 323-329. http://dx.doi.org/10.1016/S0360-3016(99)00183-2

[5] Tsujino, K., Hirota, S., Endo, M., et al. (2003) Predictive Value of Dose-Volume Histogram Parameters for Predicting Radiation Pneumonitis after Concurrent Chemoradiation for Lung Cancer. International Journal of Radiation Oncology * Biology * Physics, 55, 110-115. http://dx.doi.org/10.1016/S0360-3016(02)03807-5

[6] Rancati, T., Ceresoli, G.L., Gagliardi, G., Schipani, S. and Cattaneo, G.M. (2003) Factors Predicting Radiation Pneumonitis in Lung Cancer Patients: A Retrospective Study. Radiotherapy \& Oncology, 67, 275-283. http://dx.doi.org/10.1016/S0167-8140(03)00119-1

[7] Claude, L., Perol, D., Ginestet, C., et al. (2004) A Prospective Study on Radiation Pneumonitis Following Conformal Radiation Therapy in Non-Small-Cell Lung Cancer: Clinical and Dosimetric Factors Analysis. Radiotherapy \& Oncology, 71, 175-181. http://dx.doi.org/10.1016/j.radonc.2004.02.005

[8] Shi, A., Zhu, G., Wu, H., Yu, R., Li, F. and Xu, B. (2010) Analysis of Clinical and Dosimetric Factors Associated with Severe Acute Radiation Pneumonitis in Patients with Locally Advanced Non-Small Cell Lung Cancer Treated with Concurrent Chemotherapy and Intensity-Modulated Radiotherapy. Radiotherapy \& Oncology, 5, 35. http://dx.doi.org/10.1186/1748-717X-5-35

[9] Robnett, T.J., Machtay, M., Vines, E.F., McKenna, M.G., Algazy, K.M. and McKenna, W.G. (2000) Factors Predicting Severe Radiation Pneumonitis in Patients Receiving Definitive Chemoradiation for Lung Cancer. International Journal of Radiation Oncology * Biology * Physics, 48, 89-94. http://dx.doi.org/10.1016/S0360-3016(00)00648-9

[10] Kim, T.H., Cho, K.H., Pyo, H.R., et al. (2005) Dose-Volumetric Parameters for Predicting Severe Radiation Pneumonitis after Three-Dimensional Conformal Radiation Therapy for Lung Cancer. Radiology, 235, 208-215. http://dx.doi.org/10.1148/radiol.2351040248

[11] Yom, S.S., Liao, Z., Liu, H.H., et al. (2007) Initial Evaluation of Treatment-Related Pneumonitis in Advanced-Stage Non-Small-Cell Lung Cancer Patients Treated with Concurrent Chemotherapy and Intensity-Modulated Radiotherapy. International Journal of Radiation Oncology * Biology * Physics, 68, 94-102. http://dx.doi.org/10.1016/j.ijrobp.2006.12.031

[12] Seung, S.K. and Ross, H.J. (2009) Phase II Trial of Combined Modality Therapy with Concurrent Topotecan plus Radiotherapy Followed by Consolidation Chemotherapy for Unresectable Stage III and Selected Stage IV Non-SmallLung Cancer. International Journal of Radiation Oncology * Biology * Physics, 73, 802-809. http://dx.doi.org/10.1016/j.ijrobp.2008.04.074

[13] Rodrigues, G., Lock, M., D’Souza, D., et al. (2004) Prediction of Radiation Pneumonitis by Dose-Volume Histogram Parameters in Lung Cancer: A Systematic Review. Radiotherapy \& Oncology, 71, 127-138. http://dx.doi.org/10.1016/j.radonc.2004.02.015

[14] Curran, W., Scott, C.B., Langer, C.J., et al. (2003) Long-Term Benefit Is Observed in a Phase III Comparison of Sequential vs. Concurrent Chemo-Radiation for Patients with Unresectable Stage III NSCLC: RTOG 9410. Proceedings of American Society of Clinical Oncology, 22, 621. (Abstr)

[15] Hope, A.J., Lindsay, P.E., El Naqa, I., et al. (2006) Modeling Radiation Pneumonitis Risk with Clinical, Dosimetric, and Spatial Parameters. International Journal of Radiation Oncology * Biology * Physics, 65, 112-124. http://dx.doi.org/10.1016/j.ijrobp.2005.11.046

[16] Seppenwoolde, Y., Lebesque, J.V., De Jaeger, K., et al. (2003) Comparing Different NTCP Models That Predict the Incidence of Radiation Pneumonitis. International Journal of Radiation Oncology * Biology * Physics, 55, 724-735. http://dx.doi.org/10.1016/S0360-3016(02)03986-X

[17] Lee, J.S., Scott, C., Komaki, R., et al. (1996) Concurrent Chemoradiation Therapy with Oral Etoposide and Cisplatin for Locally Advanced Inoperable Non-Small-Cell Lung Cancer: Radiation Therapy Oncology Group Protocol 91-06. Journal of Clinical Oncology, 14, 1055-1064.

[18] Rodrigues, G., Lock, M., D’Souza, D., et al. (2004) Prediction of Radiation Pneumonitis by Dose-Volume Histogram Parameters in Lung Cancer: A Systematic Review. Radiotherapy \& Oncology, 71, 127-138. http://dx.doi.org/10.1016/j.radonc.2004.02.015

[19] Wang, S., Liao, Z., Wei, X., et al. (2006) Analysis of Clinical and Dosimetric Factors Associated with TreatmentRelated Pneumonitis (TRP) in Patients with Non-Small-Cell Lung Cancer (NSCLC) Treated with Concurrent Chemotherapy and Three-Dimensional Conformal Radiotherapy (3-DCRT). International Journal of Radiation Oncology * Biology * Physics, 66, 1399-1407. http://dx.doi.org/10.1016/j.ijrobp.2006.07.1337

[20] Byhardt, R.W., Scott, C., Sause, W.T., et al. (1998) Response, Toxicity, Failure Patterns, and Survival in Five Radia- 
tion Therapy Oncology Group (RTOG) Trials of Sequential and/or Concurrent Chemotherapy and Radiotherapy for Locally Advanced Non-Small-Cell Carcinoma of the Lung. International Journal of Radiation Oncology * Biology * Physics, 42, 469-478. http://dx.doi.org/10.1016/S0360-3016(98)00251-X

[21] Zhao, Y.H., Li, Z.P., Chen, X.M., et al. (2008) Correlation Study of Physical and Biologic Parameters to Acute Radiation Pneumonitis. Journal of Sichuan University: Medical Science Edition, 39, 796-800.

[22] Roach III, M., Gandara, D.R., You, H.S., et al. (1995) Radiation Pneumonitis Following Combined Modality Therapy for Lung Cancer: Analysis of Prognostic Factors. Journal of Clinical Oncology, 13, 2606-2612.

[23] Das, S.K., Chen, S., Deasy, J.O., et al. (2008) Combining Multiple Models to Generate Consensus: Application to Radiation-Induced Pneumonitis Prediction. Medical Physics, 35, 5098-5109. http://dx.doi.org/10.1118/1.2996012

[24] Inoue, A., Kunitoh, H., Sekine, I., et al. (2001) Radiation Pneumonitis in Lung Cancer Patients: A Study of Risk Factors and the Long-Term Prognosis. International Journal of Radiation Oncology * Biology * Physics, 49, 649-655. http://dx.doi.org/10.1016/S0360-3016(00)00783-5 